\title{
Neural signatures of compression in the retelling of past events
}

\author{
Elizabeth Musz*1 \& Janice Chen ${ }^{1}$
}

1. Department of Psychological and Brain Sciences, Johns Hopkins University,

Baltimore, MD 21218, USA

*Correspondence: emusz1@jhu.edu 


\section{Abstract}

When we retell our past experiences, we aim to reproduce some version of the original events; this reproduced version is often temporally compressed relative to the original. How does such compression of memories manifest in brain activity? One possibility is that a compressed retrieved memory manifests as a neural pattern which is more dissimilar to the original, relative to a more detailed or vivid memory. However, we argue that measuring raw dissimilarity alone is insufficient, as it confuses a variety of interesting and uninteresting changes. To address this problem, we examine brain pattern changes that are consistent across people. We show that temporal compression in individuals' retelling of past events predicts systematic encoding-torecall transformations in a number of higher associative regions. These findings elucidate how neural representations are not simply reactivated, but can also be transformed due to temporal compression during a universal form of human memory expression: verbal retelling. 


\section{Introduction}

More than any other creature, humans have a great ability to describe their past experiences to others. When we retell past experiences from memory, the goal is to reproduce some version of the original events; this reproduced version is often shorter, sparser, or otherwise reduced from the original ${ }^{1-5}$. For example, when you tell a friend about a book you recently read, your retelling is unlikely to take as long as it took you to read the book. Instead, a temporally compressed summary, in which you have extracted what you consider to be the most important aspects of the book - the main plotline, the characters' personalities, the distinctive writing style - will be much more appreciated by your friend than a verbatim recreation.

The compression of experience into memory often occurs in a similar manner across people. Psychological studies show that, given the same events, different people tend to remember and forget in patterns common with each other. One source of commonality in remembering arises from the intrinsic memorability of items: if people are asked to remember a large number of images, some images are less likely to be forgotten than others due to factors such as distinctiveness and social content ${ }^{6-12}$. In memories for more complex material such as events in a narrative or autobiographical experience, the relative prominence of event features may be modified, such that some aspects are strengthened in the remembered version while other details are minimized ${ }^{13-18}$. Individuals also draw on their world knowledge when recalling their experiences $^{13,19}$; over time, errors may be introduced that shift memories closer to existing schema $^{1,20}$, and recollections often become more semanticized, gist-like versions of the original events $^{21-25}$. Such losses and changes between encoding and recall should not necessarily be viewed failures; rather, if the changes are consistent across individuals, they may confer benefits such as building common ground needed for effective communication ${ }^{26}$.

How does the compressed nature of memories manifest in brain activity? As with behavior, the retrieved or reconstructed neural representation is an imperfect copy of the original representation ${ }^{27,28}$. Many studies have shown that, when people retrieve episodic memories, brain activity patterns similar to those present at the time of encoding a given item or event are reinstated in a set of brain regions which typically include default mode network (DMN) and high-level sensory areas, with reinstatement strength modulated by retrieval strength or vividness ${ }^{29-36}$. Unlike vivid memories that allow us to re-experience the past, "compression" suggests the initially encoded events have been altered. Thus, a compressed memory might 
present as a neural pattern during retrieval which is more dissimilar to the original pattern relative to a more detailed or vivid memory. However, retrieval conditions may differ from the encoding conditions in many ways, such as the surroundings, the task demands, and the physical state of the rememberer. Given these caveats, a raw dissimilarity measurement alone between neural patterns at encoding and those during recall seems insufficient, as it would incorporate both: 1) interesting changes, e.g., behaviorally-relevant shifts in representations of specific items or events; and 2) uninteresting changes, e.g., broad differences between brain states when one is watching video versus producing speech, as well as random decay of memory traces ${ }^{37}$. Furthermore, while raw dissimilarity captures both subject-consistent and subject-idiosyncratic changes, it cannot discriminate subject-idiosyncratic changes from task-irrelevant noise.

A complementary approach would be a test which selectively measures neural changes between encoding and recall that are consistent across people. In a prior study, Chen et al. $(2017)^{2}$ observed that activity patterns in certain brain regions changed systematically from their form during encoding — in this case, watching a movie — to an altered form during spoken recollection of the movie. Importantly, the analyses showed that whatever activity pattern change occurred between watching a given movie event (the original) and recalling it (the reproduction), the change was common across brains yet specific to individual movie events. This approach selectively identifies subject-consistent changes, separating them from state-related changes, subject-idiosyncratic changes, and task-irrelevant noise ${ }^{38}$. While raw dissimilarity between encoding and recall patterns would increase with any change, an approach which computes only subject-consistent changes necessarily rules out random decay of memory traces, i.e., identified changes are consistent across brains and thus non-random. These cross-subject, systematic pattern changes were observed in DMN cortical regions, in keeping with observations that these regions participate in episodic memory retrieval ${ }^{33}$ and carry information about mental models of situations or perspectives ${ }^{39,40}$. However, in this prior work, the neural effect was not grounded in a psychological description, e.g., not linked to any specific features of the movie contents, nor to the nature of the individuals' recollections ${ }^{2}$.

In the present study, we hypothesized that subject-consistent changes in neural patterns between encoding and retrieval—changes that are common across brains yet specific to individual events - would be related to the compression of events as participants retold them from memory. Compression, or "summarization", was assessed in the spoken recall of a recently 
viewed audiovisual movie by quantifying, for any given utterance of verbal recollection, the duration of the described event during encoding (i.e., movie-viewing). Behaviorally, we observed that speakers naturally varied the level of temporal precision that they provided for any given event. Some recollections pinpointed a specific moment (e.g., "I picked up the knife and sliced the butter"), while other descriptions summarized over longer periods of time (e.g., "I ate breakfast"), i.e., temporally compressed events to a greater degree. In neural analyses, we first asked whether summarization during recall was associated with greater raw pattern dissimilarity between encoding and recall; multi-voxel correlation analyses showed that, relative to temporally precise utterances, summary utterances during recall were indeed associated with reduced neural reinstatement in posterior medial cortex, a key DMN region, as well as weaker reductions in other posterior DMN regions. Critically, we next examined changes that were common across brains yet specific to individual movie events. As predicted, we observed that summarization was significantly positively associated with encoding-to-recall changes in a number of high-level associative and visual areas: events that were later summarized underwent greater transformation of their neural patterns between initial perception and later recall, relative to events described with high temporal precision. Our findings show that reactivation effects, typically observed in the DMN, are modulated by the degree to which people temporally compress recollected naturalistic events.

\section{Results}

\section{Description of the paradigm and dataset}

Participants $(\mathrm{n}=17)$ viewed an audiovisual stimulus, the first 50 minutes of Episode 1 of BBC's Sherlock, while undergoing fMRI. All participants reported that they had not seen any episodes of the series before. Participants were informed that immediately after viewing the video, they would be instructed to describe what they had seen. After the end of the video, participants verbally recalled the plot of the movie aloud in as much detail as possible and in their own words, also during fMRI scanning (Figure 1A). Speech was recorded with a microphone. No visual or auditory cues were provided during the recall session. Data are from Chen et al., $(2017)^{2}$. 


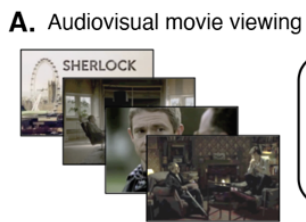

C.

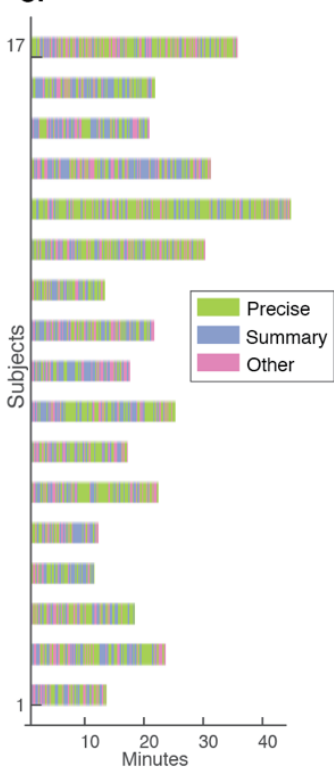

Spoken recall, no cues or audiovisual input

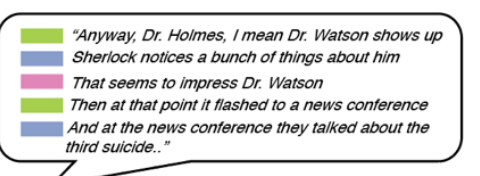
And at the news conference they talked about the
third suicide.."
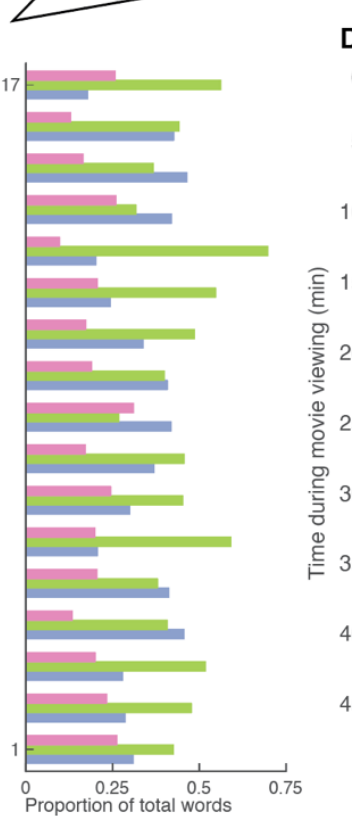

D.
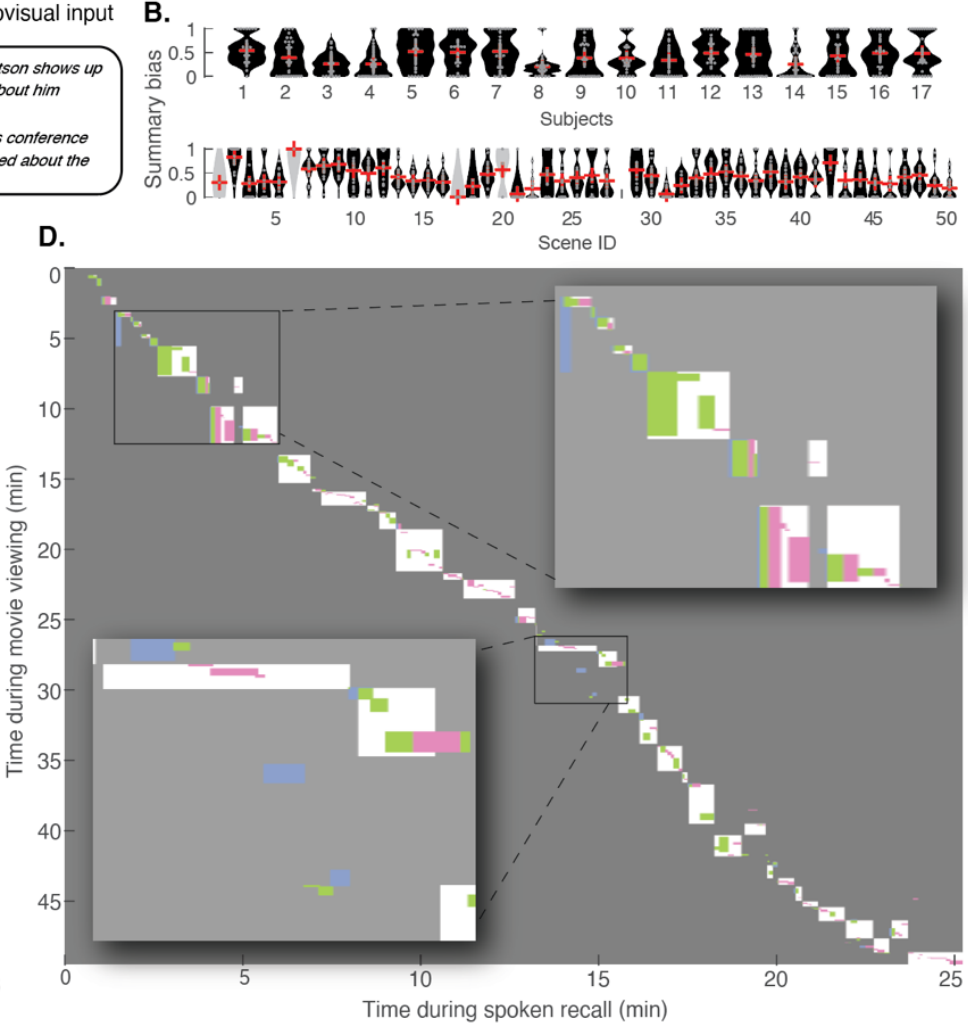

Figure 1. Experiment design and recall behavior. A) Left: During Run 1, participants watched a 50-minute movie, BBC's Sherlock, Episode 1. Right: During the immediately following Run 2, participants recalled the movie content via verbal recall. Participants' responses were audio recorded, transcribed, and segmented into utterances that roughly aligned with ends of sentences and breaks in speech (each row). Each utterance was categorized according to the movie content that it referred to, and according to its temporal precision (i.e., the amount of time that it took for the described events to elapse in the movie). Utterances that described events that occurred in ten seconds or less were coded as "Temporally Precise," while events that spanned longer than ten seconds were coded as "Summary." Utterances were coded as "Other" if they did not provide literal and accurate descriptions of the movie events. B) Violin plots depicting the Summary bias across scenes for each individual subject (top row) and across subjects for each individual scene (bottom). Scenes shown in grey were recalled by less than $5 / 17$ participants and were excluded from all subsequent inter-subject analyses. C) Left: Subject-specific timelines of recall utterances, colored by degree of temporal precision. Right: Proportion of spoken words during recall that were included in utterances coded as Summary, Precise, or Other. D) Diagram of scene durations during movie viewing (y-axis) and movie recall (x-axis) and scene order during recall (diagonal) in a representative participant. Each white box shows one scene from the original 50 -scene segmentation ${ }^{2}$. Overlaid on top are the durations and scene identities of the specific recall utterances, coded by temporal precision. The right and left insets show zoomed-in subsets of this recall behavior. For illustration purposes only, "Other" recall utterances are labeled as the most recently described movie scene.

\section{Classification of summarization vs. temporal precision for individual utterances during spoken}

recall

To characterize summarization during recall, we created a rubric to score individual utterances spoken by participants during their verbal recall of the movie. The rubric assesses the degree to which a given utterance refers to a specific moment of the movie (Temporally Precise), 
as opposed to describing a longer period of time (Summary). Here, utterances correspond roughly to sentences; in continuous speech it is often not clear how to delineate sentences, and thus transcriptionists were instructed to break the text into short sentences using their best judgment. Each participant's speech was labeled sentence-by-sentence by two coders. Summarization was calculated in three ways: "Direct judgment", "Automatic", and "Temporal compression factor". As the three yielded similar results, in the remainder of the paper we report results derived from the "Direct judgment" procedure: utterances were classified as Temporally Precise if they described movie events that elapsed in less than ten seconds and classified as Summary if they described events spanning more than ten seconds during the movie. See Methods for further details about the rubric and comparisons between all three approaches.

The total duration of the recall session varied across participants, as did the total number of recall utterances, ranging from 114 to 468 utterances $(M=203.1, S D=110.1)$. Each utterance was comprised of 14 words on average $(S D=2.4)$, and the mean utterance duration was $6.83 \mathrm{~s}$ $(S D=1.6 \mathrm{~s})$. On average, $46.7 \%$ of participants' utterances were coded as Temporally Precise and $29.8 \%$ as Summary. Across participants an average of $23.6 \%$ of utterances were labeled as "Other" (statements that were inaccurate or not about specific movie events) and excluded from subsequent analyses (Figure 1). To compare the relative amounts of the different speech categories present in each subject's recall, we counted the number of words included in each category, summing across all of a subject's statements (Figure 1C). Most participants produced a greater proportion of Temporally Precise versus Summary words (11/17 participants), although the number of words and the relative proportion of utterances in the different categories varied across individuals. For additional descriptive statistics see Supplementary Table 2.

\section{Summary utterances diverge from the original movie contents: a text-based analysis}

Summarization entails modifying the original event content, e.g., by synthesizing multiple elements of, or selectively recalling only some aspects of, the original experience. As a validation test for our rubric, we sought to verify that recall utterances classified as Summary diverged more from the original material than those classified as Temporally Precise. To this end, we performed a text-based analysis using natural language processing tools to estimate similarity between movie events and recall utterances. 
The movie content was transcribed and annotated; then, these detailed natural language descriptions of the movie events were segmented into 1000 unique "micro-segments", each approximately $4 \mathrm{~s}$ in duration $(S D=2.2 \mathrm{~s}, \min =1 \mathrm{~s}, \max =19 \mathrm{~s})$, and then the recall utterances for each subject were linked to the matching movie micro-segment from a written description of the movie (see ${ }^{2,41}$ for details). After converting each of the movie micro-segment annotations and recall utterances into a unique 512-element sentence embedding vector using the Universal Sentence Encoder ${ }^{42}$, we computed similarity between each recall utterance vector and all movie segment vectors for each participant. Movie vs. recall text cosine similarity was reliably greater for matching versus mismatching movie micro-segments and recall utterances; this was true for both Summary utterances, $t(16)=32.54, p<0.001(M$ cosine difference $=0.10, S D=0.01)$ and Temporally Precise utterances, $t(16)=44.77, p<0.001,(M$ cosine difference $=0.18, S D=0.02)$. Critically, movie vs. recall text cosine similarity was significantly lower for micro-segments that were later summarized, relative to micro-segments that were precisely recalled, $t(16)=8.35, p<$ $0.001(M$ cosine difference $=0.07, S D=0.04)$; this effect was in the same direction for all individual subjects (17 of 17) (Supplementary Figure 1). These results provide support for the effectiveness of our rubric in separating utterances which compressed across longer periods of movie time (Summary) from those which described events Temporally Precise manner.

To what extent did Summary utterances and Temporally Precise utterances arise from recall of distinct events? In other words, did a participant tend to recall one set of events in a summarized manner and a different set of events in a temporally precise manner? For each subject, each Summary utterance's micro-segments were compared against the micro-segments associated with each Temporally Precise utterance. On average, 30\% of a subject's Summary utterances covered micro-segments that were also described during Temporally Precise utterances $(S D=17.3, \min =7.7 \%, \max =74 \%)$.

Encoding-Recall neural pattern similarity is weaker for summarized than for temporally precise utterances

We next examined the strength of neural reactivation, i.e., raw pattern similarity, associated with Summary utterances as opposed to Temporally Precise utterances. By design, Summary utterances are associated with greater temporal compression of the memory relative to 
the encoded material; and as revealed above in the text-based analysis, Summary utterances diverge more from the original movie contents in terms of sentence-level semantics. Thus, we predicted that Summary utterances should be accompanied by weaker reactivation (greater raw pattern dissimilarity) than Temporally Precise utterances.

To measure memory reactivation, we computed the correlation between a given subject's brain pattern while 1) viewing specific moments of the movie versus 2) recalling the same moments. In order to separate the conditions of Summary and Temporally Precise to the greatest possible extent, this analysis was performed at the micro-segment level, i.e., separately for movie micro-segments that were later summarized versus those that were later described in a temporally precise manner.

The reinstatement analysis (movie vs. recall pattern correlation) was conducted first in a posterior medial cortex (PMC) ROI motivated by the previous study ${ }^{2}$, and then in cortical parcels across the whole brain (citations and see Methods). Reinstatement was significant in the PMC ROI both for movie micro-segments that were recalled in a Temporally Precise manner, $t(16)=$ 9.49, $p<.001$ (mean $r=.10, S D=.04$ ) and those that were later Summarized, $t(16)=7.07, p<.001$ (mean $r=.05, S D=.03$ ). Furthermore, in 16/17 subjects, reinstatement was greater for Temporally Precise than for Summarized recall; $t(16)=5.11, p<0.001$, two-tailed tests (Figure 2A). In the whole-brain parcel-based analysis, several regions exhibited significant reinstatement for both the Temporally Precise (Figure 2B) and Summarized (Figure 2C) micro-segments, including multiple parcels in lateral prefrontal and lateral temporal cortex, as well as posterior medial areas. The plurality of regions exhibiting these effects were located in parcels within the DMN (as defined from the parcellation atlas; see Figure 3B), including 36\% of the parcels in the Temporally Precise reinstatement map, and 30\% in the Summarized reinstatement map (Table 1). These results are consistent with reinstatement effects reported in the searchlight analysis of the same data ${ }^{2}$ as well as with many observations in the literature using a variety of stimuli and retrieval methods ${ }^{29-31}$.

Several parcels in the DMN showed numerically greater reactivation for Temporally Precisely recalled versus Summarized movie micro-segments, including left lateral parietal cortex and posterior medial cortex (Figure 2D); however for this contrast no parcels survived FDR correction at $q=0.05$. See Figure $3 \mathrm{~B}$ and Supplemental Table 3 for lists of the parcels defined as DMN and PMC, respectively. 


\begin{tabular}{|c|c|c|c|c|c|c|c|c|c|}
\hline Parcel map & $\begin{array}{c}\text { Relevant } \\
\text { Figure }\end{array}$ & $\begin{array}{c}\text { Total } \\
\text { parcels }\end{array}$ & Default & $\begin{array}{l}\text { Fronto- } \\
\text { temporal }\end{array}$ & Visual & $\begin{array}{c}\text { Ventral } \\
\text { Attention }\end{array}$ & $\begin{array}{c}\text { Dorsal } \\
\text { Attention }\end{array}$ & $\begin{array}{c}\text { Somato- } \\
\text { motor }\end{array}$ & Limbic \\
\hline $\begin{array}{l}\text { 1. Precise } \\
\text { Reactivation }\end{array}$ & $\begin{array}{l}\text { Figure } \\
\text { 2B }\end{array}$ & 207 & $0.36(75)$ & $0.18(38)$ & $\begin{array}{l}0.15 \\
(30) \\
\end{array}$ & $0.14(29)$ & $0.12(24)$ & $0.03(7)$ & $\begin{array}{c}0.02 \\
(4) \\
\end{array}$ \\
\hline $\begin{array}{l}\text { 2. Summary } \\
\text { Reactivation }\end{array}$ & $\begin{array}{c}\text { Figure } \\
2 \mathrm{C}\end{array}$ & 243 & $0.30(73)$ & $0.19(45)$ & $\begin{array}{l}0.13 \\
(32) \\
\end{array}$ & $0.16(39)$ & $0.10(25)$ & $\begin{array}{l}0.08 \\
(20) \\
\end{array}$ & $\begin{array}{c}0.04 \\
(9)\end{array}$ \\
\hline $\begin{array}{l}\text { 3. Precise }> \\
\text { Summary } \\
\text { Reactivation }\end{array}$ & $\begin{array}{l}\text { Figure } \\
2 \mathrm{D}\end{array}$ & 14 & $0.71(10)$ & 0 & 0 & $0.07(1)$ & $0.21(3)$ & 0 & 0 \\
\hline $\begin{array}{l}\text { 4. Summary > } \\
\text { Precise } \\
\text { Reactivation }\end{array}$ & $\begin{array}{l}\text { Figure } \\
2 \mathrm{D}\end{array}$ & 2 & 0 & 0 & 0 & 0 & 0 & $1(2)$ & 0 \\
\hline $\begin{array}{l}\text { 5. Memory } \\
\text { Transformation }\end{array}$ & $\begin{array}{c}\text { Figure } \\
3 \mathrm{~A}\end{array}$ & 136 & $0.29(39)$ & $0.15(21)$ & $\begin{array}{l}0.21 \\
(28) \\
\end{array}$ & $0.13(18)$ & $0.11(15)$ & $0.07(9)$ & $\begin{array}{c}0.04 \\
(6)\end{array}$ \\
\hline $\begin{array}{l}\text { 6. Summary } \\
\text { Bias \& } \\
\text { Memory } \\
\text { Transformation }\end{array}$ & $\begin{array}{l}\text { Figure } \\
4 \mathrm{~B}\end{array}$ & 41 & $0.27(11)$ & $0.20(8)$ & $\begin{array}{c}0.22 \\
(9)\end{array}$ & $0.20(8)$ & $0.07(3)$ & $0.05(2)$ & 0 \\
\hline
\end{tabular}

Table 1. For each map, above-threshold parcels were distributed across the seven functional networks identified in Yeo et al., 2011 (see Figure 3B). Each row lists the proportion (and count) of parcels located in each network. Analyses corresponding to rows 1-2 were tested in all 400 parcels. Rows 3-4 were limited to parcels that showed reliable effects in either row 1 or row 2 (283 total parcels tested); row 5 was limited to parcels where either encoding-to-recall or recall-to-recall was reliable (174 total parcels); and row 6 was limited to parcels that showed reliable effects in row 5 (136 total parcels). Results were thresholded at the $p<.05$ level, except for rows 3-4 ( $p<.10)$.

In sum, Summary utterances during recall were associated with weaker neural reinstatement in PMC, relative to Temporally Precise statements, and a subsequent brain-wide analysis found a number of DMN parcels in medial and lateral parietal cortex trending in the same direction. These results suggest that temporal compression during recall modulates reinstatement effects in DMN subregions. Whereas previous work has reported that DMN reinstatement can vary depending on subjective ratings of memory vividness ${ }^{34,36}$, here we show that the temporal compression of movie events, as identified in individuals' naturally varying speech content during spoken recall, is associated with decreased pattern similarity between encoding and recall. 

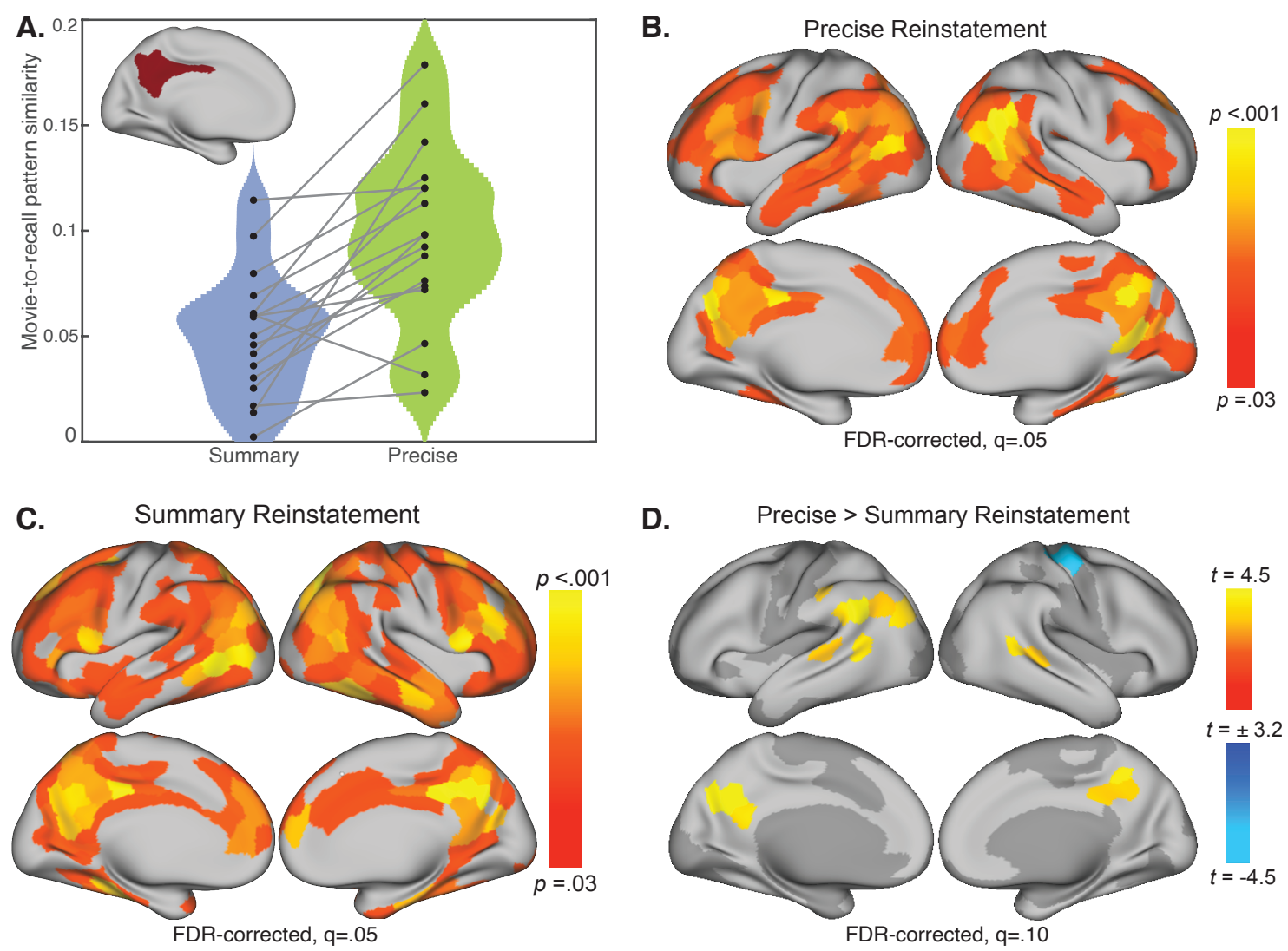

Figure 2. Within-subject movie-recall reinstatement analysis for movie "micro-segments" later recalled in a Summarized versus Temporally Precise manner. Pattern similarity was performed at the individual-subjects level using movie "micro-segments" of short duration $(M=4 \mathrm{~s}, S D=2.2 \mathrm{~s})$. A) Reinstatement analysis in the bilateral PMC ROI. Movie-recall pattern similarity was greater for movie micro-segments recalled in a Temporally Precise as opposed to a Summarized manner, $t(16)=5.11, p<0.001$, two-tailed. B) Parcel map of movie versus recall pattern similarity, limited to movie segments that were later recalled in a Temporally Precise manner. C) Parcel map of movierecall pattern similarity, limited to movie micro-segments that were later Summarized during recall. D) Parcels where movie-recall similarity was greater for Temporally Precisely recalled segments versus Summarized segments. This analysis was limited to parcels that showed reliable reinstatement effects in either the Temporally Precise (2B) or Summary (2C) reinstatement maps; excluded parcels are shown in dark grey.

Regions exhibiting the memory "transformation" effect

We hypothesized that subject-consistent changes in neural patterns between encoding and retrieval — changes that are common across brains yet specific to individual events — would be related to the behaviorally-identified compression of retrieved events. Thus, we next identified brain parcels that exhibited cross-subject-consistent changes between encoding and retrieval, i.e., a "transformation" effect. This analysis reiterates an analysis conducted in Chen et al. (2017) now using a parcel-based approach rather than a searchlight procedure. 
Chen et al. (2017) $)^{2}$ introduced a simple method for identifying functionally relevant changes between encoding and recall patterns by leveraging similarity between individuals. In brief, the reasoning was that if memory-evoked patterns are more similar between individuals than to the original event pattern, then it can be inferred that patterns were transformed between perception and memory in a systematic (non-noise) manner. Using this method, encoding-torecall transformation was observed in many subregions of the default mode network in a searchlight analysis.

A. Encoding-to-Recall Transformation

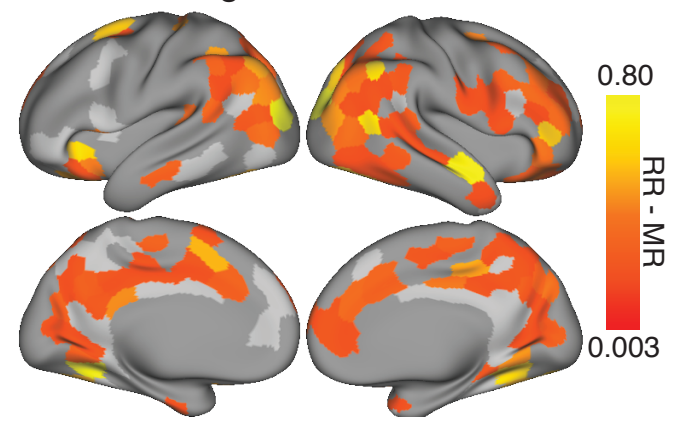

B. Schaefer et al. (2018) Parcellation

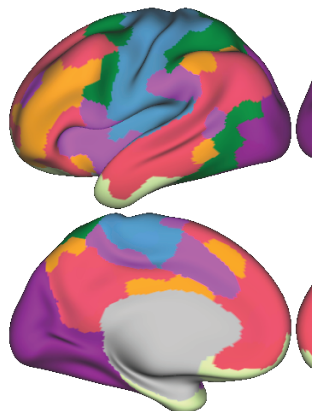

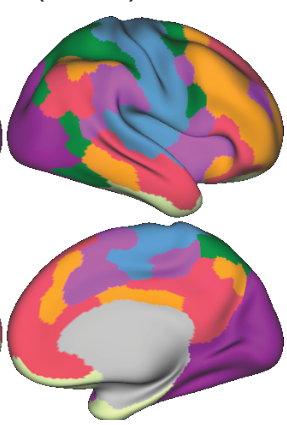

Default

Ventral Attention

Limbic

Frontoparietal

Somatomotor

Dorsal Attention

Visual

Figure 3. A) Parcel map depicting group-level memory transformation effects, where scene-level recall-to-recall similarity (RR) was greater than movie-to-recall similarity (MR) across subjects (136/174 parcels). Values were only computed in parcels within a "reliability mask," which was limited to parcels where either RR similarity or MR similarity was reliable (174/400 parcels). Parcels outside of this reliability mask are shown in dark grey, and parcels in the reliability mask where RR did not exceed MR are shown in light grey. Subsequent analyses were limited to parcels that exhibited memory transformation (all colored parcels). B) All reported similarity values were computed in 400 parcels from an independent whole-brain resting-state parcellation ${ }^{43}$. Each parcel is associated with one of seven functional networks ${ }^{43,44}$.

For the present study, we employed a parcel-based approach, and thus our first step was to recalculate transformation within individual parcels $\left(400\right.$ parcels $\left.^{43}\right)$. The brain was first masked to retain only parcels that exhibited reliable activity patterns across subjects during movie-viewing or recall (174/400 parcels, see Methods). Within this mask, transformation scores were computed by subtracting each parcel's movie-recall correlation from its recall-recall correlation value. Transformation scores were positive in 136 of 174 parcels and these were retained for subsequent analyses (Figure 3A). These parcels were distributed throughout several networks, including DMN (29\% of parcels), the Visual Network (21\% of parcels), the Frontoparietal Network (15\% of parcels), and the Dorsal (13\%) and Ventral (11\%) Attention Networks (see Figure 3B and Table 1). A comparison to the searchlight-based transformation map from Chen et al. $(2017)^{2}$ is provided in Supplementary Figure 2. 
Summarization during recall scales positively with memory transformation

We next sought to test our hypothesis that compression, as identified by summarization behavior in recollection speech, would be positively related to transformation - the magnitude of cross-subject-consistent pattern change from encoding to subsequent recall. We first computed scene-level "Summary bias" scores for all participants, as transformation was calculated at the 50 -scene level in the prior study (in contrast to the 1000 micro-segment level we used in the reinstatement analysis reported above in Figure 2). "Summary bias" was computed, for each movie scene, as the proportion of words describing that scene during recall that were from Summary utterances, versus Temporally Precise utterances. While the movie scenes varied in duration ( $M=37.3$ seconds, $S D=27.8)$, scene duration was not correlated with Summary bias across participants, $t(16)=-0.3, p=0.76$ (mean $r=-0.01, S D=0.16$ ). Participants varied in the degree to which they summarized each movie scene (Figure 1B). Across movie scenes, the relative prominence of summarization does not appear to systematically differ depending on the movie timeline, although most participants summarize the second movie scene and produce temporally precise utterances about the final scene (Figure 1B).

To test whether a bias toward summarization was positively related to memory transformation across movie scenes, we computed the correlation between these variables in each brain parcel. Testing was constrained to parcels that exhibited positive memory transformation scores in the prior analysis (i.e., parcels where recall-recall similarity exceeded movie-recall similarity; see Figure 3A), as our interpretation of negative memory transformation scores is that subject-consistent encoding-to-recall changes have not been detected. We found a positive relationship between Summary bias and memory transformation in a number of parcels, such that scenes with a greater Summary bias underwent greater pattern changes from movie to recall, relative to scenes with a greater bias toward being recalled in Temporally Precise manner (Figure 4B): 40 parcels passed FDR correction at $\mathrm{q}=0.05$. These parcels were distributed across several functional networks: $27 \%$ of parcels were located in the DMN, $22 \%$ in the Visual Network, and 20\% each in the Dorsal Attention Network or Frontoparietal Network (Figure 4C and Table 1). Of note, transformation increased with greater Summary bias in the precuneus, retrosplenial cortex, bilateral lateral parietal cortex, and right prefrontal cortex. The effect was 
significant in the reverse direction for two parcels, located in left medial temporal cortex and right ventral temporal cortex; i.e., for these two parcels, scenes with lower Summary bias scores exhibited stronger memory transformation.

A. Memory transformation vs. Summary bias in a given parcel

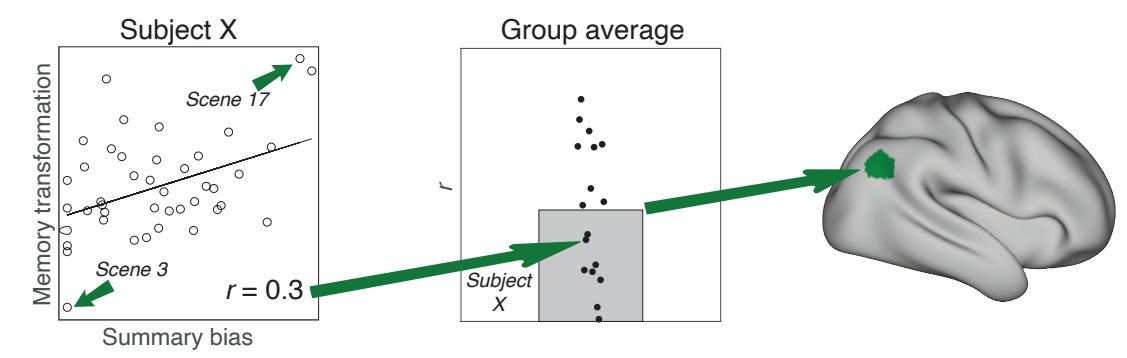

B.

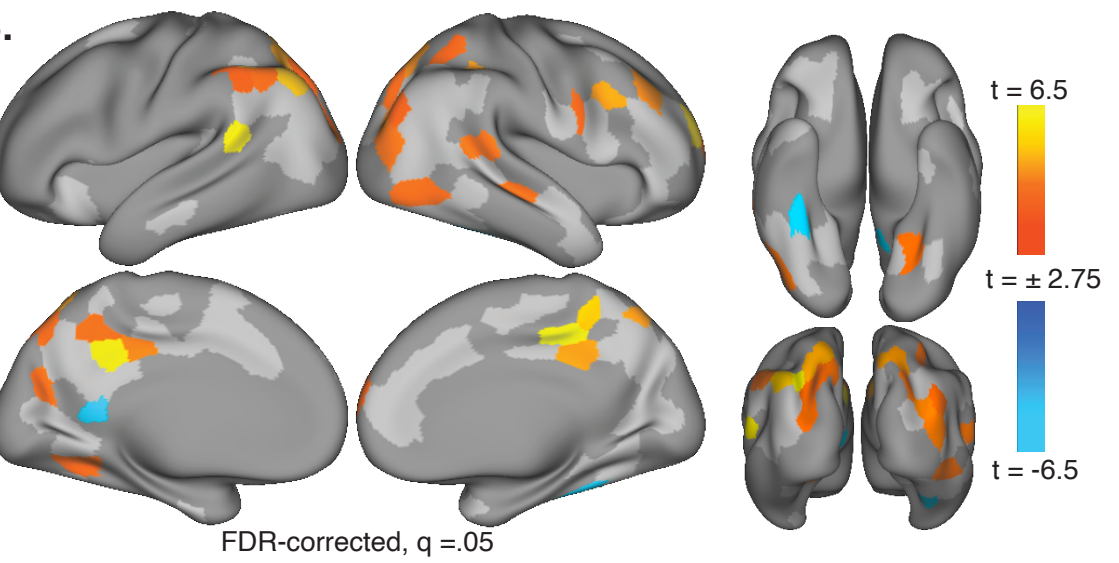

C.

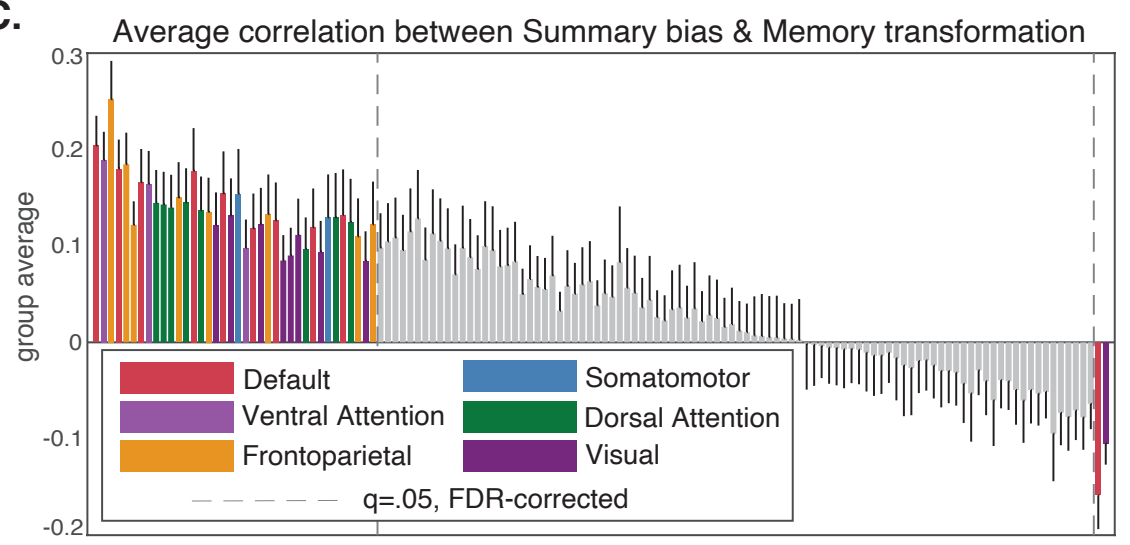

Figure 4. A) Analysis schematic depicting how memory transformation is related to summarization in each participant and each tested brain parcel. In each parcel, the scene-level transformation values are correlated with each individual subject's Summary bias scores. The example scatterplot (left) depicts the correlation between Summary bias and degree of memory transformation across scenes for one subject. Subject-level correlation values are then submitted to a random effects analysis ( $t$-test versus zero, two-tailed). The bar plot (middle) depicts the correlation values, aggregated across subjects. The resulting t-statistic value is then assigned to the parcel on the brain map (right). B) Parcel-level map of the relationship between memory transformation and summarization. This relationship was only tested in parcels that showed reliable memory transformation effects (136/400 parcels, see Figure 3A). Memory transformation scaled with summarization in 40/136 parcels (colored). In 96/136 of tested parcels, this relationship was not statistically reliable (light grey). 264/400 parcels were outside the memory transformation mask and were 
excluded from analysis (dark grey). C) Relationship between summarization and memory transformation across the 136 memory transformation parcels. Bar color indicates the functional network of each above-threshold parcel. Error bars indicate standard error of the mean.

\section{Discussion}

Our memories of the past often seem to be temporally compressed versions of the original experiences. In this study, we investigated how the brain instantiates compression of memories for naturalistic events. We hypothesized that reactivation effects during spoken retelling, previously observed in many high-level associative and visual areas, would be modulated by the degree to which people temporally compress experienced events during their recall. To test this idea, we first developed a rubric to score individual utterances spoken by participants during their unguided verbal recall of a recently viewed movie. The rubric assesses the degree to which a given utterance refers to a specific moment of the movie (Temporally Precise), as opposed to describing a longer period of time (Summarizing). We found that Summary utterances during recall were associated with greater dissimilarity between encoding and recall in PMC, and to a lesser extent other regions of the DMN, relative to Temporally Precise statements. This observation agrees with prior studies showing that reinstatement (encoding-recall similarity) is positively related to subjective vividness ${ }^{36}$ and the amount of detail of the retrieved information ${ }^{45,46}$. Our results extend these findings by showing that naturally varying speech content — in particular, signatures of temporal compression during recall—also modulate reinstatement effects. However, we argue that raw dissimilarity alone is an incomplete measure, as it incorporates both interesting changes (e.g., behaviorally-relevant shifts in representations of specific items or events) and uninteresting changes (e.g., broad differences between brain states at encoding versus at recall, and random decay). Thus, in a complementary analysis, we tested the relationship between summarization during recall and the encoding-torecall "transformation" of brain activity patterns - a measure which selectively identifies changes which are consistent across subjects ${ }^{2}$. The results showed that, at the scene level, a bias toward summarization in recall speech significantly predicted the magnitude of transformation in a number of high-level visual and associative areas, with a plurality of parcels found in the DMN. Importantly, as the changes were shared across subjects, this result demonstrates that temporal compression during recall does not merely result in noisier reinstatement; rather, neural 
representations of events shift between encoding and recall in a systematic, potentially meaningful way. Together, these findings elucidate how temporal compression of memories for naturalistic events manifests in brain activity.

The act of recollecting an event from memory typically takes less time than the original event duration ${ }^{1,3,4,47}$. Why should memory of real-world events be a compressed version of the original? In addressing this question, it is important to distinguish between compression of the stored information and of the retrieved information. In the current study, we measured brain activity during encoding and during retrieval; this allowed us to test ideas about retrieved information but did not give us access to the representational format of information stored in memory. Compression makes intuitive sense as a strategy for efficient storage - there is no need to remember everything, only what is required to meet future demands ${ }^{48}$. By analogy, computer algorithms for compressing image files succeed by discarding information that is not needed, while still meeting the demand that the compressed image will appear similar to the original ${ }^{49}$. In the case of JPEG, for example, as the human eye discriminates image brightness more finely than color, compression involves downsampling color information while brightness information is retained at higher resolution.

On top of what has been modified in the stored memory representation, the demands of a given task can lead to further compression in the retrieved information: e.g., telling someone the steps of a recipe versus describing the atmosphere of the cooking class where you learned the recipe. Prior work has demonstrated that episodic memory retrieval can be directed or biased by the person's task demands and goals ${ }^{14,50}$. In the case of our task, the instructions were to retell the story (the plot) in as much detail as possible, "as if telling a friend". Thus, people were mainly conveying the plot of the story and some pertinent sensory features. If subjects had been probed with questions demanding more detail, most likely they would be able to produce more detail $^{51}$. However, the current task did not require that; instead, participants naturally varied the level of detail they provided across utterances. Our Temporal precision and Summary scores, and related behavioral measures which estimate descriptive precision during recall ${ }^{52,53}$ indicate only the level of detail retrieved at the time of the test, not of the stored information. Future work using memory probes which more exhaustively probe stored memories could investigate the gap between what is stored and what is retrieved. 
Why should the compression scheme be similar across people? We suggest three reasons. First, compression of stored or retrieved memories could be similar across people simply because brain anatomy and function are similar across people. In comparative studies, learning behavior for a given task is more variable across species than within species ${ }^{54}$. Second, beyond shared anatomy, participants also have shared past experiences to some degree; several studies have shown that shared past experiences lead to shared neural responses to narrative stimuli in DMN regions. For example, Parkinson and colleagues (2018) showed that inter-subject similarity in posterior medial cortex during movie-viewing was predicted by proximity in a social network, which the authors attribute to homophily: friends are likely to have similar prior experiences ${ }^{55}$. However, extensive shared past is not needed to predict variation in inter-subject similarity: Yeshurun and colleagues (2017) showed that, when subjects were presented with an ambiguous story, a single disambiguating paragraph presented just before the story was sufficient to push subjects into distinct groups ${ }^{56}$. Thus, a follow-up question for the current study is: given that people evince some similarity in how they compress memories of events, to what extent is this influenced by shared prior experiences? Finally, a third reason that memories might follow a similar compression scheme across people is that such commonality could facilitate communication between individuals. Given a shared experience by a group of individuals, later conversation between the individuals may be more coherent if they have a common ground ${ }^{57,58}$ based on similarly remembered, forgotten, or otherwise transformed memories. This point may be particularly pertinent in our paradigm, as participants recounted their memories orally, a delivery format that may have evoked a mode of thinking associated with inter-personal communication.

What is the problem with raw dissimilarity as a measure of compression, and what is provided by the complementary "transformation" measure? It has been widely demonstrated that episodic remembering is accompanied by "reinstatement" in a set of brain regions which typically include default mode network and high-level sensory areas; reinstatement is calculated as a similarity score between the brain activity pattern at encoding versus at retrieval ${ }^{32}$. As "compression" implies that retrieval will be different in some way from encoding, a natural prediction would be that the more compressed a retrieved memory is, the more dissimilar its neural representation should be to that observed during encoding. Indeed, we did see this relationship in PMC (Figure 2). However, a raw dissimilarity measurement is problematic 
because it will be driven up by multiple types of non-signal which cannot be discriminated from each other: broad differences between brain states at encoding vs. at retrieval, random decay of memory traces during the delay between encoding and retrieval, and other measurement or machine noise ${ }^{37}$. Dissimilarity will also be driven up by both subject-consistent and subjectidiosyncratic changes, both of which are interesting signals which experimenters would like to measure; but again, these cannot be separated from task-irrelevant noise. Thus, we applied a complementary test which selectively measures neural changes between encoding and recall ${ }^{2}$ to selectively identify subject-consistent changes, separating them from state-related changes, subject-idiosyncratic changes, and task-irrelevant noise.

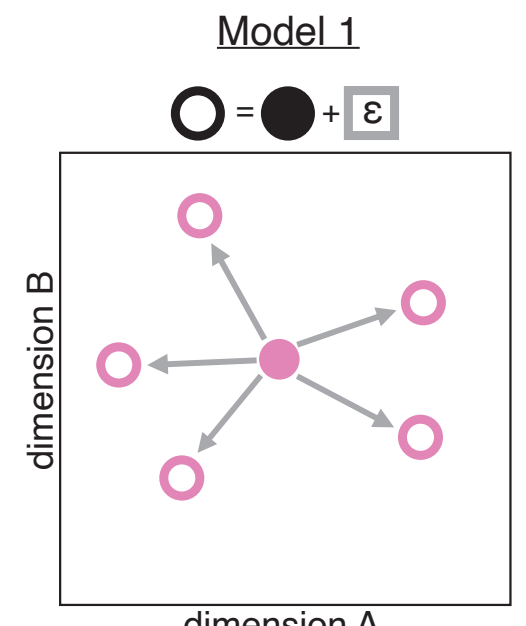

$\operatorname{dimension} \mathrm{A}$

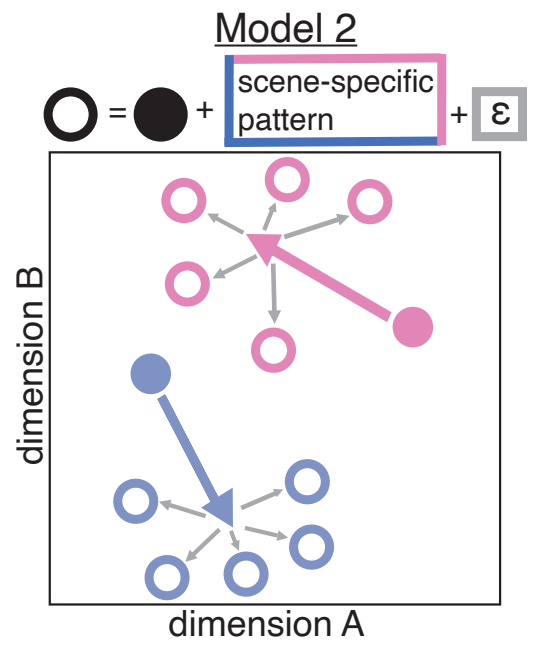

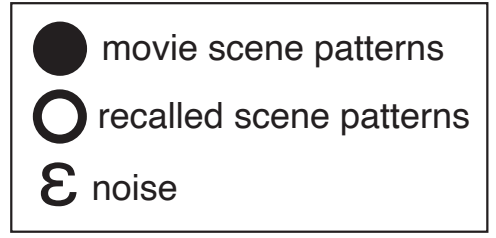

Figure 5. Schematic of possible contributions to the pattern changes observed between encoding and recall of a movie scene. Each solid dot represents the neural pattern evoked by a single scene during movie viewing, here shown in a hypothetical two-dimensional space. The rings of the same color depict the neural patterns evoked when different subjects recall the scene. In both proposed models, a scene's pattern change from movie to recall is partly due to noise or subject-idiosyncratic shifts, shown as gray arrows. In Model 1, the pattern shifts from movie to recall are entirely explained by either noise or subject-idiosyncratic shifts (the two are indistinguishable). In Model 2, the pattern change is consistent across people, but varies by movie scene, such that magnitude and direction of the shift (colored arrows) depends on the features of each movie scene. Noise/subject-idiosyncratic shifts (gray arrows) are also present.

Chen et al. (2017) $)^{2}$ reasoned that if retrieved activity patterns are more similar between individuals than to the originating pattern at encoding, then it can be inferred that patterns were transformed in the interval between encoding and retrieval in a systematic (non-noise) manner. In the schematic shown in Figure 5, brain activity patterns are visualized as vectors in multidimensional space, where each dimension corresponds to a brain voxel; we propose functional labels for different types of pattern shifts. 
- Noise and subject-idiosyncratic shifts. For a given event, the retrieved event pattern may differ from the original event pattern due to 1) task-irrelevant noise, and/or 2) the idiosyncratic way in which an individual recollects the event; 1 and 2 cannot be distinguished. In this scenario (Figure 5, Model 1), the direction in which the "recall" pattern shifts away from the original "movie" pattern varies across individuals. Similarity between different individuals' recalled scene patterns must be lower than similarity between recall and movie patterns (in Figure 5, Model 1, the average distance between rings must be greater than the average distance between the rings and the filled circle).

- Event-specific shifts. Alternatively, the retrieved event pattern may differ from the original event pattern in a common way across people, such that each person's recall pattern shifts away from the original pattern in the same direction for a given event, but in different directions for different events (Figure 5, Model 2). With the addition of this cross-subjectconsistent shift, it becomes possible for recall patterns to be more similar to each other than they are to the original patterns (in Figure 5 Model 2, for a given color, average distance between rings is smaller than the average distance between the rings and the filled circle). These event-specific shifts reflect differences in the stimulus features across events that in turn affect their transformation (one component of which could be compression) between encoding and recall. For example, imagine that for Event $\mathrm{A}$ the most memorable features are foreground objects, while for event B the most memorable feature is spatial layout; and that $50 \%$ of voxels are sensitive to object category, while the other $50 \%$ are sensitive to spatial layout. In this case, the A and B event patterns would shift in different directions, but the direction of the shift would be the same across people within each event (A or B).

In the current paper, we show evidence that event-specific shifts are predicted by behaviorallyexpressed summarization, or temporal compression, during recall; the degree of compression determines the degree of observed pattern transformation in a number of parcels throughout high-level visual and associative areas, with a plurality of parcels located in the DMN.

Although these inter-subject comparisons enable identification of pattern changes that are shared across subjects, they do not capture pattern changes that are specific to individual subjects, which includes both a subject-specific signal component and a subject-specific noise component. Recently, there has been growing interest in measuring such subject-idiosyncratic 
differences using other methods such as inter-subject representational similarity analysis (ISRSA) ${ }^{59-63}$. Using IS-RSA, researchers can examine whether second order statistics among individuals (e.g., their similarity relations along a behavioral dimension) correspond to the geometric mapping of brain pattern similarities across individuals. For example, while watching an animation of abstract shapes ${ }^{62}$ and listening to an audio story ${ }^{64}$, subject pairs who expressed more similar interpretations of the stimulus also showed increased ISC in several brain regions. A promising avenue of exploration for future work could be the quantification of separate contributions for subject-shared and subject-idiosyncratic signals during naturalistic encoding and recollection.

Changes in event representations between encoding and recall, whether they are subjectidiosyncratic or subject-consistent, are likely not limited to compression. We envision compression as an operation wherein representations are selectively pruned, and perhaps guided by previously learned templates or schemas ${ }^{1}$. Alongside the compression of the encoded experience, "new" information could also be incorporated into the retrieved memory, e.g., the representation could be integrated or supplemented with related prior episodes ${ }^{65-68}$. Such changes would also contribute to event-specific shifts (see Figure 5: changes could be modeled as new information being added to recall event patterns in a subject-consistent manner) but would not be captured in our Summary/Temporally Precise ratings. In the current study, we focused on compression due to what we observed in the behavior: on average, $76 \%$ of participants' utterances were judged by raters to be literal and accurate (either Summary or Temporally precise), leaving $24 \%$ of utterances in the "Other" category, which included inaccurate and metacognitive statements, as well as elaborations and inferences which might be considered "new" information. Exploration of additional operations embedded in the changes between neural event representations at encoding and recall could be pursued with paradigms in which subjects are specifically instructed, e.g., to compress, integrate, or add information during retrieval $^{69}$.

In conclusion, this work provides evidence that the temporal compression of remembered events manifests in brain activity in a distributed set of high-level visual and associative areas. During temporally compressed recall, individuals' speech content and brain patterns showed decreased similarity to the original movie events, indicating that the summarized memories are altered versions of the initial experiences. In order to address possible problems with raw 
dissimilarity measures, we additionally assessed encoding-to-recall pattern changes that were consistent across subjects; even under these restricted conditions we observed a significant relationship between temporal compression and neural pattern changes in many of the same high-level regions, with a plurality of the identified parcels falling within the DMN. These findings illuminate how our memories for real-world events diverge from the original experiences in a consistent manner across people, and reveal the neural transformations that accompany summarization or compression of our experiences.

\section{Methods}

\section{Participants}

Twenty-two adults (10 female, ages 18-26) were recruited from the Princeton community. All participants were right-handed native English speakers who reported normal or corrected-to-normal vision and had not watched any episodes of Sherlock prior to the experiment. Participants provided written consent prior to experiment in accordance with the Princeton University Institutional Review Board. Data from five participants were discarded due to excessive head motion, short recall (less than ten minutes), or falling asleep during scanning. This dataset was originally reported in Chen et al., $(2017)^{2}$.

\section{Movie event boundaries}

The movie (Sherlock Episode 1) was segmented into individual events at two different levels of granularity. For the coarse-grained segmentation, the movie was split into 50 scenes, where scene boundaries followed major narrative shifts ${ }^{2}$. Coarse scenes were on average $57.7 \mathrm{~s}$ long $(S D=41.6 \mathrm{~s}, \max =180 \mathrm{~s}, \min =11 \mathrm{~s})$. The fine-grained segmentation consisted of 1,000 "micro-segments," including the original 50-scene boundaries. Fine-grained micro-segments lasted 3.95 seconds on average $(S D=2.20 \mathrm{~s}, \max =19 \mathrm{~s}, \min =1 \mathrm{~s})$.

\section{Utterance Coding}

Each participant's speech from the recall session was transcribed. The transcribed speech was then divided into individual utterances, where each utterance roughly corresponded to a single complete sentence. For long sentences that contained three or more subordinate clauses, 
sentences were broken up into multiple utterances, where the utterance boundaries were defined by pauses (e.g., implied commas) in the participant's speech. Each utterance was comprised of 14 words on average $(S D=2.4)$, and the mean utterance duration was $6.83 \mathrm{~s}(S D=1.6 \mathrm{~s})$. The total duration the recall session varied across participants ${ }^{2}$, as did the total number of recall utterances, ranging from 114 to 468 utterances across participants (average $=203.1, S D=110.1$ ). For additional descriptive statistics see Supplementary Table 2.

Each utterance was coded according to 1) the movie events it described and 2) the amount of time that it took for the described events to elapse in the movie (i.e., the degree of temporal precision). First, for the movie scene coding, each utterance was assigned a "start movie segment" and "end movie segment" label. The scene labels were assigned according to the fine-grained, 1000 micro-segmentation. The labeling of each utterance's starting and ending movie scenes was completed by a single annotator with no knowledge of the experimental design or hypotheses ${ }^{2}$.

Next, for temporal precision, each utterance was labeled as either "Summary," "Temporally Precise" or "Other" (Figure 1). These labels were generated in two distinct ways: Direct Judgment and Automatic. The two methods yield consistent labels across subjects, $\mathrm{r}=0.60$ (min: $\mathrm{r}=0.39$, $\max : \mathrm{r}=0.77, S D=0.12$ ). In addition, the automated labeling method can provide a continuous measure of temporal compression, as opposed to the binary summary/temporally precise distinction. The main reported results use the Direct Judgment labels; we describe the procedure for generating these labels below.

For the direct judgment labeling, participants' speech during recall was categorized into the three non-overlapping categories (i.e., Summary, Temporally Precise, and Other). Literal and accurate statements about the movie content were categorized as Temporally Precise if they describe movie events that elapsed in less than ten seconds. In contrast, literal and accurate statements were classified as Summary if they described events that took longer than ten seconds to occur. Only literal and accurate utterances were coded; utterances that contained factually incorrect information (i.e., confabulation) or that referred to events other than the movie content (i.e., not recall) were coded as "Other" and excluded from subsequent analysis.

The temporal precision labeling was completed by two coders (including author EM) who were provided the definitions for Temporally Precise and Summary utterances listed above. Each coder worked separately to label all utterances according to these definitions. After 
completing the labels for each participant, the two coders met and identified all utterances for which their labels did not match. The raters originally had a 77\% agreement rate. For utterances with mismatching labels, the coders then reviewed the utterance content and re-watched the corresponding movie scenes together to arrive at a consensus label.

All participants produced utterances in all three categories (Figure 1C). On average, 47\% of participants' utterances were coded as Temporally Precise and 30\% as Summary. Across participants an average of $24 \%$ of utterances were labeled as "Other" and excluded from subsequent analyses (Figure 1).

To compare the relative amounts of the different speech categories present in each subject's recall, we counted the number of words included in each category, summing across all of a subject's statements (Figure 1B). Most participants produced a greater proportion of Temporally Precise versus Summary words (11/17 participants), although the number of words and the relative proportion of utterances in the different categories varied across individuals.

In contrast to the Direct Judgment method, the Automatic method considers the microsegment(s) assigned to each recall utterance, and sums the duration of these segments to determine whether each utterance's described movie events elapsed in less than or greater than ten seconds. Additional details for the Automatic method, and details for measuring temporal compression as a continuous measure (termed "temporal compression factor"), are provided in the Supplementary Materials.

Text-based analysis of the similarity between movie annotations and summary and precise recall

In order to compute the overlap between movie and recall content, and to test how movieto-recall text similarity varied by recall type (i.e., for Summary versus Temporally Precise recall utterances), we performed an utterance-level analysis comparing the speech generated at recall against the original movie annotations. All movie segment annotations and recall statements were converted into unique 512-element vectors using the Universal Sentence Encoder algorithm ${ }^{42}$. We computed the similarity between each recall statement vector and its corresponding movie segment vector(s). If a recall utterance described multiple segments, a separate cosine similarity score was computed for each of these segments and then averaged together to yield one composite cosine similarity score for the utterance. Average movie versus recall vector similarity was then computed separately for each participant and each recall type 
(i.e., movie segments vs. Summary utterances and movie segments vs. Precise utterances). To test for significance within each condition (i.e., for Summary and Precise separately), paired $t$ tests (two tailed) were performed to compare cosine similarity for vectors of matching segments (i.e., movie segments and recall utterances that covered the same content) versus mismatching segments. To directly compare participants' average movie-to-recall cosine similarity for Summary versus Temporally Precise recall segments, the cosine similarity values for the matching segments of each recall type were also submitted to a paired $t$-test (two-tailed).

\section{Within-Subjects Reinstatement for Summarized Versus Temporally Precise Recall}

Memory reactivation was measured at the individual-subjects level by computing pattern similarity between each micro-segment during movie viewing and subsequent recall utterance(s) that described each micro-segment. If several recall utterances described the same microsegment, the patterns for these utterances were first averaged together prior to computing the reinstatement value. Reactivation scores were calculated separately for segments that were later recalled in a Temporally Precise manner versus Summarized. If a micro-segment was later described with both Summary and Temporally Precise utterances, it received two separate reactivation scores.

For the ROI-based analysis, pattern similarity was computed in voxels located in a bilateral PMC ROI (see Figure 3A and Supplementary Table 2 for ROI definition). The resulting reinstatement values across subjects were submitted to a one-sample t-test versus zero (two tailed), computed separately for Summary and Temporally Precise Reactivation. To directly compare reinstatement across recall type, each subject's two reactivation values were submitted to a paired t-test (two tailed).

In a parcel-based analysis, reinstatement was computed separately for Summary and Temporally Precise recall in every parcel in the brain. To determine statistical significance at each parcel, one-tailed $p$-values were computed by comparing the group-average reinstatement value to a null distribution that was generated by randomly shuffling the segment-to-utterance correlation matrices 1000 times. To determine thresholding, the parcel-level p-values were submitted to FDR correction ( $q=0.05)$. Statistical significance and FDR correction were computed separately for the Summary reinstatement and Temporally Precise reinstatement maps. 
To create a contrast map of Summary versus Temporally Precise reinstatement at the parcel level, we limited our analyses to parcels that showed a reliable Summary or Temporally Precise reinstatement effect (283/400 parcels, see Figure 3D). At each parcel included in this mask, we directly compared the two reinstatement values across subjects with a paired $t$-test (two tailed). Subsequent FDR correction was limited to parcels included in this mask. No parcels survived FDR correction at $\mathrm{q}=0.05$; at a more liberal threshold $(\mathrm{q}=0.10)$, ten parcels showed marginal effects of greater reinstatement for Temporally Precise versus Summarized recall.

\section{Computing Degree of Summarization by Movie Scene}

We computed the average degree of temporal precision for each movie scene in each subject. First, for each participant, we sorted the utterances according to the movie scene(s) that they referred to. Note that the same utterance could be assigned to several different movie scenes, depending on its "start movie scene" and "stop movie scene" labels. Then, we counted the total number of words spoken about each movie scene by summing across all words in all of its corresponding utterances. We then sorted each scene's utterances by temporal precision, and removed "stop" words (e.g., "a", "is", "the”) and non-words (e.g., "uh", "um”) from all utterance transcriptions (see Supplementary Table 1). Finally, we counted the proportion of words that were assigned each label (i.e., Summary, Temporally Precise, or Other). For each participant, this analysis yielded one \%Summary bias score for each movie scene that the participant recalled. Movie scenes that were recalled by less than five out of the 17 participants were excluded from this analysis.

\section{Memory transformation: Methods (parcels)}

We tested for brain areas that contain scene-specific content that is shared across individuals in each of 400 parcels from an independent whole-brain resting state parcellation ${ }^{43}$. In each parcel, we first performed the scene-level, between-subject analyses, computing recallto-recall similarity and movie-to-recall similarity for each scene ${ }^{2}$. The movie-to-recall analysis uses the fMRI data acquired during both the movie viewing session and the subsequent free recall session. Each timepoint was labeled according to the scene that the subject was currently viewing (during encoding) or describing (during recall). The constituent TRs in each scene were then averaged together, yielding 50 scene patterns for the movie data in each subject. For the 
recall data, the number of scene patterns varied across subjects, depending on which scenes each subject mentioned during recall. For the between-subjects movie-to-recall analysis, each individual's movie scene patterns were compared to the corresponding recall patterns for that same scene, averaged across all of the other subjects who recalled that scene. This analysis yield two values for each parcel: one indexing the scene-average movie-to-recall similarity in the parcel, and one for the scene-average recall-to-recall similarity. For each comparison, statistical significance was determined by shuffling the scene labels 1000 times to generate to a null distribution. A one-tailed p-value was calculated as the proportion of values from the null distribution that were equal to or greater than the observed similarity values for matching scenes. We then constrained our analyses to parcels that showed significant effects in either the movieto-recall or recall-to-recall comparisons. Of the 400 parcels, 174 met this criterion (all light gray and hot colored parcels in Figure 3). For these "reliable" parcels, we identified the ones where between-subjects recall-to-recall similarity exceeded movie-to-recall similarity. The difference between these values quantifies the memory transformation value for that parcel.

\section{Linking Transformation \& Summarization: Methods}

To test for relationships between degree of summarization and memory transformation, we focused on the parcels that showed memory transformation effects. In these parcels, we correlated each subject's scene-level \%Summary bias scores with the group-average memory transformation value for each scene. Across subjects, we tested for reliable correlations between $\%$ Summary bias and memory transformation values with a one-sample t-test versus zero (twotailed) performed at each included parcel. Across parcels, we used FDR correction $(\mathrm{p}<0.05$, corrected) to determine statistical thresholding. 


\section{Data Availability}

Data and materials are available on Open Science Framework at: https://osf.io/8gmes/

These materials include data and analysis codes for reproducing the parcel maps displayed in Figure 3A and Figure 4B; the Summary/Precise Rubric used to produce the Direct Judgments of temporal precision; and utterance-level temporal precision labels for the transcribed recall data for all participants.

\section{Acknowledgments}

We thank James Antony, Buddhika Bellana, and Christopher Honey for their comments on an earlier version of the manuscript. We thank Qingwei Zhang for assistance with behavioral ratings and Yoonjung Lee for her feedback on the data and analysis materials available on the Open Science Framework. This work was supported by a National Science Foundation Postdoctoral Research Fellowship (SBE SPRF 1911650 to E.M.) and a Sloan Research Fellowship (to J.C.).

\section{Author Contributions}

E.M. and J.C. conceived and designed the experiments. E.M. and J.C. performed the experiments and analyzed the data. E.M. and J.C. wrote and edited the paper.

\section{Citation Diversity Statement}

Recent work in several fields of science has identified a bias in citation practices such that papers from women and other minority scholars are under-cited relative to the number of such papers in the field ${ }^{70-78}$. Here we sought to proactively consider choosing references that reflect the diversity of the field in thought, form of contribution, gender, race, ethnicity, and other factors. First, we obtained the predicted gender of the first and last author of each reference by using databases that store the probability of a first name being carried by a woman ${ }^{74,79}$. By this measure (and excluding self-citations to the first and last authors of our current paper), our references contain 16.2\% woman(first)/woman(last), 12.92\% man/woman, 24.42\% woman/man, 
and $46.45 \% \mathrm{man} / \mathrm{man}$. This method is limited in that a) names, pronouns, and social media profiles used to construct the databases may not, in every case, be indicative of gender identity and b) it cannot account for intersex, non-binary, or transgender people. Second, we obtained predicted racial/ethnic category of the first and last author of each reference by databases that store the probability of a first and last name being carried by an author of color ${ }^{80,81}$. By this measure (and excluding self-citations), our references contain $8.14 \%$ author of color (first)/author of color(last), 15.43\% white author/author of color, $18.88 \%$ author of color/white author, and $57.56 \%$ white author/white author. This method is limited in that a) names, Census entries, and Wikipedia profiles used to make the predictions may not be indicative of racial/ethnic identity, and b) it cannot account for Indigenous and mixed-race authors, or those who may face differential biases due to the ambiguous racialization or ethnicization of their names. We look forward to future work that could help us to better understand how to support equitable practices in science. 


\section{References}

1. Bartlett, F. C. Remembering: A study in experimental and social psychology. (Cambridge University Press, 1932).

2. Chen, J. et al. Shared memories reveal shared structure in neural activity across individuals. Nat Neurosci 20, 115-125 (2017).

3. Jeunehomme, O., Folville, A., Stawarczyk, D., Van der Linden, M. \& D’Argembeau, A. Temporal compression in episodic memory for real-life events. Memory 26, 759-770 (2018).

4. Kashima, Y. Maintaining Cultural Stereotypes in the Serial Reproduction of Narratives. Pers Soc Psychol Bull 26, 594-604 (2000).

5. Michelmann, S., Staresina, B. P., Bowman, H. \& Hanslmayr, S. Speed of time-compressed forward replay flexibly changes in human episodic memory. (2018) doi:10.1101/323774.

6. Bainbridge, W. A., Isola, P. \& Oliva, A. The intrinsic memorability of face photographs. Journal of Experimental Psychology: General 142, 1323-1334 (2013).

7. Bainbridge, W. A., Dilks, D. D. \& Oliva, A. Memorability: A stimulus-driven perceptual neural signature distinctive from memory. NeuroImage 149, 141-152 (2017).

8. Bylinskii, Z., Isola, P., Bainbridge, C., Torralba, A. \& Oliva, A. Intrinsic and extrinsic effects on image memorability. Vision Research 116, 165-178 (2015).

9. Isola, P., Xiao, J., Torralba, A. \& Oliva, A. What makes an image memorable? in CVPR $20118(2011)$

10. Parikh, D., Isola, P., Torralba, A. \& Oliva, A. Understanding the intrinsic memorability of images. Journal of Vision 12, 1082-1082 (2012). 
11. Shepherd, J. W., Gibling, F. \& Ellis, H. D. The effects of distinctiveness, presentation time and delay on face recognition. European Journal of Cognitive Psychology 3, 137-145 (1991).

12. Vokey, J. R. \& Read, J. D. Familiarity, memorability, and the effect of typicality on the recognition of faces. Memory \& Cognition 20, 291-302 (1992).

13. Bonasia, K. et al. Prior knowledge modulates the neural substrates of encoding and retrieving naturalistic events at short and long delays. Neurobiology of Learning and Memory (2018) doi:10.1016/j.nlm.2018.02.017.

14. Loftus, E. F. \& Hoffman, H. G. Misinformation and Memory: The Creation of New Memories. Journal of Experimental Psychology: General 118, 100-104 (1989).

15. Morrow, D. G., Bower, G. H. \& Greenspan, S. L. Updating situation models during narrative comprehension. Journal of Memory and Language 28, 292-312 (1989).

16. Pezdek, K. Event memory and autobiographical memory for the events of September 11, 2001. Appl. Cognit. Psychol. 17, 1033-1045 (2003).

17. Schmolck, H., Buffalo, E. A. \& Squire, L. R. Memory Distortions Develop Over Time: Recollections of the O.J. Simpson Trial Verdict After 15 and 32 Months. Psychol Sci 11, 3945 (2000).

18. Sekeres, M. J. et al. Recovering and preventing loss of detailed memory: differential rates of forgetting for detail types in episodic memory. Learn. Mem. 23, 72-82 (2016).

19. Bird, C. M., Davies, R. A., Ward, J. \& Burgess, N. Effects of pre-experimental knowledge on recognition memory. Learning \& Memory 18, 11-14 (2010).

20. Hollingworth, H. L. The Central Tendency of Judgment. The Journal of Philosophy, Psychology and Scientific Methods 7, 461 (1910). 
21. Alba, J. W. \& Hasher, L. Is memory schematic? Psychological Bulletin 93, 203-231 (1983).

22. Baldassano, C., Hasson, U. \& Norman, K. A. Representation of Real-World Event Schemas during Narrative Perception. J. Neurosci. 38, 9689-9699 (2018).

23. Bower, G. H. \& Clark-Meyers, G. Memory for scripts with organized vs. randomized presentations. British Journal of Psychology 71, 369-377 (1980).

24. Ghosh, V. E. \& Gilboa, A. What is a memory schema? A historical perspective on current neuroscience literature. Neuropsychologia 53, 104-114 (2014).

25. Sachs, J. S. Recognition memory for syntactic and semantic aspects of connected discourse. Perception \& Psychophysics 2, 437-442 (1967).

26. Clark, H. H., Schreuder, R. \& Buttrick, S. Common ground at the understanding of demonstrative reference. Journal of Verbal Learning and Verbal Behavior 22, 245-258 (1983).

27. Hassabis, D. \& Maguire, E. A. Deconstructing episodic memory with construction. Trends in Cognitive Sciences 11, 299-306 (2007).

28. Zadbood, A., Chen, J., Leong, Y. C., Norman, K. A. \& Hasson, U. How We Transmit Memories to Other Brains: Constructing Shared Neural Representations Via Communication. Cerebral Cortex 27, 4988-5000 (2017).

29. Johnson, J. D., McDuff, S. G. R., Rugg, M. D. \& Norman, K. A. Recollection, Familiarity, and Cortical Reinstatement: A Multivoxel Pattern Analysis. Neuron 63, 697-708 (2009).

30. Kuhl, B. A. \& Chun, M. M. Successful Remembering Elicits Event-Specific Activity Patterns in Lateral Parietal Cortex. Journal of Neuroscience 34, 8051-8060 (2014).

31. Polyn, S. M. Category-Specific Cortical Activity Precedes Retrieval During Memory Search. Science 310, 1963-1966 (2005). 
32. Ritchey, M., Wing, E. A., LaBar, K. S. \& Cabeza, R. Neural Similarity Between Encoding and Retrieval is Related to Memory Via Hippocampal Interactions. Cerebral Cortex 23, $2818-2828$ (2013).

33. Rugg, M. D. \& Vilberg, K. L. Brain networks underlying episodic memory retrieval. Current Opinion in Neurobiology 23, 255-260 (2013).

34. Sreekumar, V., Nielson, D. M., Smith, T. A., Dennis, S. J. \& Sederberg, P. B. The experience of vivid autobiographical reminiscence is supported by subjective content representations in the precuneus. Sci Rep 8, 14899 (2018).

35. St-Laurent, M., Abdi, H., Bondad, A. \& Buchsbaum, B. R. Memory Reactivation in Healthy Aging: Evidence of Stimulus-Specific Dedifferentiation. Journal of Neuroscience 34, 41754186 (2014).

36. St-Laurent, M., Abdi, H. \& Buchsbaum, B. R. Distributed Patterns of Reactivation Predict Vividness of Recollection. Journal of Cognitive Neuroscience 27, 2000-2018 (2015).

37. Favila, S. E., Lee, H. \& Kuhl, B. A. Transforming the Concept of Memory Reactivation. Trends in Neurosciences 43, 939-950 (2020).

38. Nastase, S. A., Gazzola, V., Hasson, U. \& Keysers, C. Measuring shared responses across subjects using intersubject correlation. Social Cognitive and Affective Neuroscience nsz037 (2019) doi:10.1093/scan/nsz037.

39. Ranganath, C. \& Ritchey, M. Two cortical systems for memory-guided behaviour. Nat Rev Neurosci 13, 713-726 (2012).

40. Ritchey, M. \& Cooper, R. A. Deconstructing the Posterior Medial Episodic Network. Trends in Cognitive Sciences 24, 451-465 (2020). 
41. Vodrahalli, K. et al. Mapping between fMRI responses to movies and their natural language annotations. NeuroImage 180, 223-231 (2018).

42. Cer, D. et al. Universal Sentence Encoder. arXiv:1803.11175 [cs] (2018).

43. Schaefer, A. et al. Local-Global Parcellation of the Human Cerebral Cortex from Intrinsic Functional Connectivity MRI. Cerebral Cortex 28, 3095-3114 (2018).

44. Thomas Yeo, B. T. et al. The organization of the human cerebral cortex estimated by intrinsic functional connectivity. Journal of Neurophysiology 106, 1125-1165 (2011).

45. Leiker, E. K. \& Johnson, J. D. Neural reinstatement and the amount of information recollected. Brain Research 1582, 125-138 (2014).

46. Wing, E. A., Ritchey, M. \& Cabeza, R. Reinstatement of Individual Past Events Revealed by the Similarity of Distributed Activation Patterns during Encoding and Retrieval. Journal of Cognitive Neuroscience 27, 679-691 (2015).

47. Jeunehomme, O. \& D'Argembeau, A. Event segmentation and the temporal compression of experience in episodic memory. Psychological Research 84, 481-490 (2020).

48. Schacter, D. L., Norman, K. A. \& Koutstaal, W. THE COGNITIVE NEUROSCIENCE OF CONSTRUCTIVE MEMORY. Annu. Rev. Psychol. 49, 289-318 (1998).

49. Haines, R. The effects of video compression on acceptability of images for monitoring life sciences experiments. vol. 3239 (National Aeronautics and Space Administration, Office of Management, Scientific and Technical Information Program., 1992).

50. Lahnakoski, J. M. et al. Synchronous brain activity across individuals underlies shared psychological perspectives. NeuroImage 100, 316-324 (2014).

51. Goldsmith, M., Koriat, A. \& Weinberg-Eliezer, A. Strategic regulation of grain size memory reporting. Journal of Experimental Psychology: General 131, 73-95 (2002). 
52. Antony, J. W. et al. Behavioral, Physiological, and Neural Signatures of Surprise during Naturalistic Sports Viewing. Neuron 109, 377-390.e7 (2021).

53. Heusser, A. C., Fitzpatrick, P. C. \& Manning, J. R. Geometric models reveal behavioural and neural signatures of transforming experiences into memories. Nat Hum Behav (2021) doi:10.1038/s41562-021-01051-6.

54. Frick, K. M., Stillner, E. T. \& Berger-Sweeney, J. Mice are not little rats: species differences in a one-day water maze task. NeuroReport 11, 3461-3465 (2000).

55. Parkinson, C., Kleinbaum, A. M. \& Wheatley, T. Similar neural responses predict friendship. Nat Commun 9, 332 (2018).

56. Yeshurun, Y. et al. Same Story, Different Story: The Neural Representation of Interpretive Frameworks. Psychol Sci 28, 307-319 (2017).

57. Brown-Schmidt, S. \& Duff, M. C. Memory and Common Ground Processes in Language Use. Top Cogn Sci 8, 722-736 (2016).

58. H.H. Clark \& Wilkes-Gibbs, D. Definite reference and mutual knowledge. in Elements of discourse understanding (eds. Joshi, A. K., Webber, B. \& Sag, I. A.) 10-63 (Cambridge University Press, 1981).

59. Chen, P.-H. A., Jolly, E., Cheong, J. H. \& Chang, L. J. Intersubject representational similarity analysis reveals individual variations in affective experience when watching erotic movies. NeuroImage 216, 116851 (2020).

60. Finn, E. S., Corlett, P. R., Chen, G., Bandettini, P. A. \& Constable, R. T. Trait paranoia shapes inter-subject synchrony in brain activity during an ambiguous social narrative. Nat Commun 9, 2043 (2018). 
61. Finn, E. S. et al. Idiosynchrony: From shared responses to individual differences during naturalistic neuroimaging. NeuroImage 215, 116828 (2020).

62. Nguyen, M., Vanderwal, T. \& Hasson, U. Shared understanding of narratives is correlated with shared neural responses. NeuroImage 184, 161-170 (2019).

63. van Baar, J. M., Chang, L. J. \& Sanfey, A. G. The computational and neural substrates of moral strategies in social decision-making. Nat Commun 10, 1483 (2019).

64. Saalasti, S. et al. Inferior parietal lobule and early visual areas support elicitation of individualized meanings during narrative listening. Brain Behav 9, e01288 (2019).

65. Bellana, B., Mansour, R., Ladyka-Wojcik, N., Grady, C. L. \& Moscovitch, M. The influence of prior knowledge on the formation of detailed and durable memories. Journal of Memory and Language 121, 104264 (2021).

66. Keidel, J. L., Oedekoven, C. S. H., Tut, A. C. \& Bird, C. M. Multiscale Integration of Contextual Information During a Naturalistic Task. Cerebral Cortex 28, 3531-3539 (2018).

67. Raykov, P. P., Keidel, J. L., Oakhill, J. \& Bird, C. M. Activation of Person Knowledge in Medial Prefrontal Cortex during the Encoding of New Lifelike Events. Cerebral Cortex 31, 3494-3505 (2021).

68. van Kesteren, M. T. R., Fernandez, G., Norris, D. G. \& Hermans, E. J. Persistent schemadependent hippocampal-neocortical connectivity during memory encoding and postencoding rest in humans. Proceedings of the National Academy of Sciences 107, 7550-7555 (2010).

69. Richter, F. R., Cooper, R. A., Bays, P. M. \& Simons, J. S. Distinct neural mechanisms underlie the success, precision, and vividness of episodic memory. eLife 5, e18260 (2016). 
70. Bertolero, M. A. et al. Racial and ethnic imbalance in neuroscience reference lists and intersections with gender. http://biorxiv.org/lookup/doi/10.1101/2020.10.12.336230 (2020) doi:10.1101/2020.10.12.336230.

71. Caplar, N., Tacchella, S. \& Birrer, S. Quantitative evaluation of gender bias in astronomical publications from citation counts. Nat Astron 1, 0141 (2017).

72. Chatterjee, P. \& Werner, R. M. Gender Disparity in Citations in High-Impact Journal Articles. JAMA Netw Open 4, e2114509 (2021).

73. Dion, M. L., Sumner, J. L. \& Mitchell, S. M. Gendered Citation Patterns across Political Science and Social Science Methodology Fields. Polit. Anal. 26, 312-327 (2018).

74. Dworkin, J. D. et al. The extent and drivers of gender imbalance in neuroscience reference lists. Nat Neurosci 23, 918-926 (2020).

75. Fulvio, J. M., Akinnola, I. \& Postle, B. R. Gender (Im)balance in Citation Practices in Cognitive Neuroscience. Journal of Cognitive Neuroscience 33, 3-7 (2021).

76. Maliniak, D., Powers, R. \& Walter, B. F. The Gender Citation Gap in International Relations. Int Org 67, 889-922 (2013).

77. Mitchell, S. M., Lange, S. \& Brus, H. Gendered Citation Patterns in International Relations Journals. Int Stud Perspect 14, 485-492 (2013).

78. Wang, X. et al. Gendered citation practices in the field of communication. Annals of the International Communication Association 45, 134-153 (2021).

79. Zhou, Dale et al. Gender Diversity Statement and Code Notebook v1.0. (Zenodo, 2020). doi:10.5281/ZENODO.3672110.

80. Ambekar, A., Ward, C., Mohammed, J., Male, S. \& Skiena, S. Name-ethnicity classification from open sources. in Proceedings of the 15th ACM SIGKDD international conference on 
Knowledge discovery and data mining - KDD ’09 49 (ACM Press, 2009).

doi:10.1145/1557019.1557032.

81. Sood, G. \& Laohaprapanon, S. Predicting Race and Ethnicity From the Sequence of Characters in a Name. arXiv:1805.02109 [stat] (2018). 


\section{Supplementary Materials}

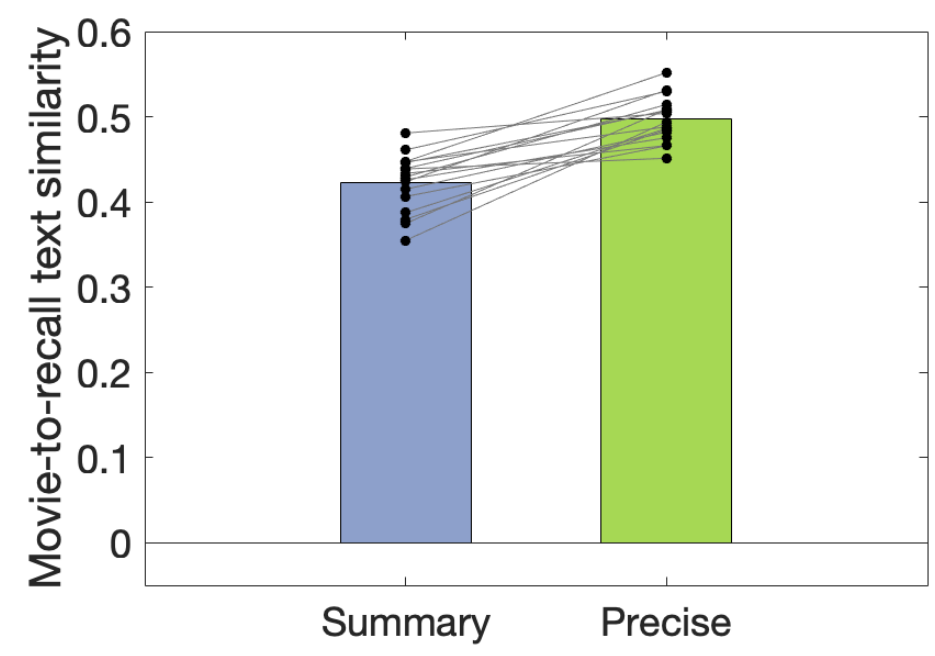

Supplementary Figure 1. Cosine similarity between text embeddings derived from movie annotations versus spoken recall, separated by recall type for each subject. Movie-to-recall text cosine similarity was separately computed for summary versus precise recall utterances.

\begin{tabular}{|l|l|l|l|}
\hline & All utterances & Precise & Summary \\
\hline $\begin{array}{l}\text { Number of } \\
\text { utterances }\end{array}$ & $203.1(110.1), 114-468$ & $108.1(75.6), 36-280$ & $54.3(15.7), 34-91$ \\
\hline $\begin{array}{l}\text { Number of words } \\
\text { per utterance }\end{array}$ & $13.87(2.39), 10.2-19.5$ & $13.57(2.1), 10.1-18.0$ & $15.75(3.34), 10.8-22.1$ \\
\hline $\begin{array}{l}\text { Duration per } \\
\text { utterance (seconds) }\end{array}$ & $6.83(1.6), 4.4-10.0$ & $6.29(1.31), 4.1-8.7$ & $8.21(2.2), 4.9-12.2$ \\
\hline $\begin{array}{l}\text { Proportion of all } \\
\text { utterances }\end{array}$ & $0.47(0.1), 0.3-0.71$ & $0.3(0.1), 0.16-0.40$ \\
\hline
\end{tabular}

Supplementary Table 1. Descriptive statistics of recall utterances. Entries list the group average value, the standard deviation in parentheses, and the minimum to maximum range of values across subjects.

Supplementary Table 2. List of stop words excluded from word counts of transcribed recall speech: a, an, and, are, as, at, be, by, for, from, has, in, is, its, of, on, that, the, to, was, were, will, with, uh, um, uhm, umm, uhh 


\section{Encoding-to-Recall Transformation}
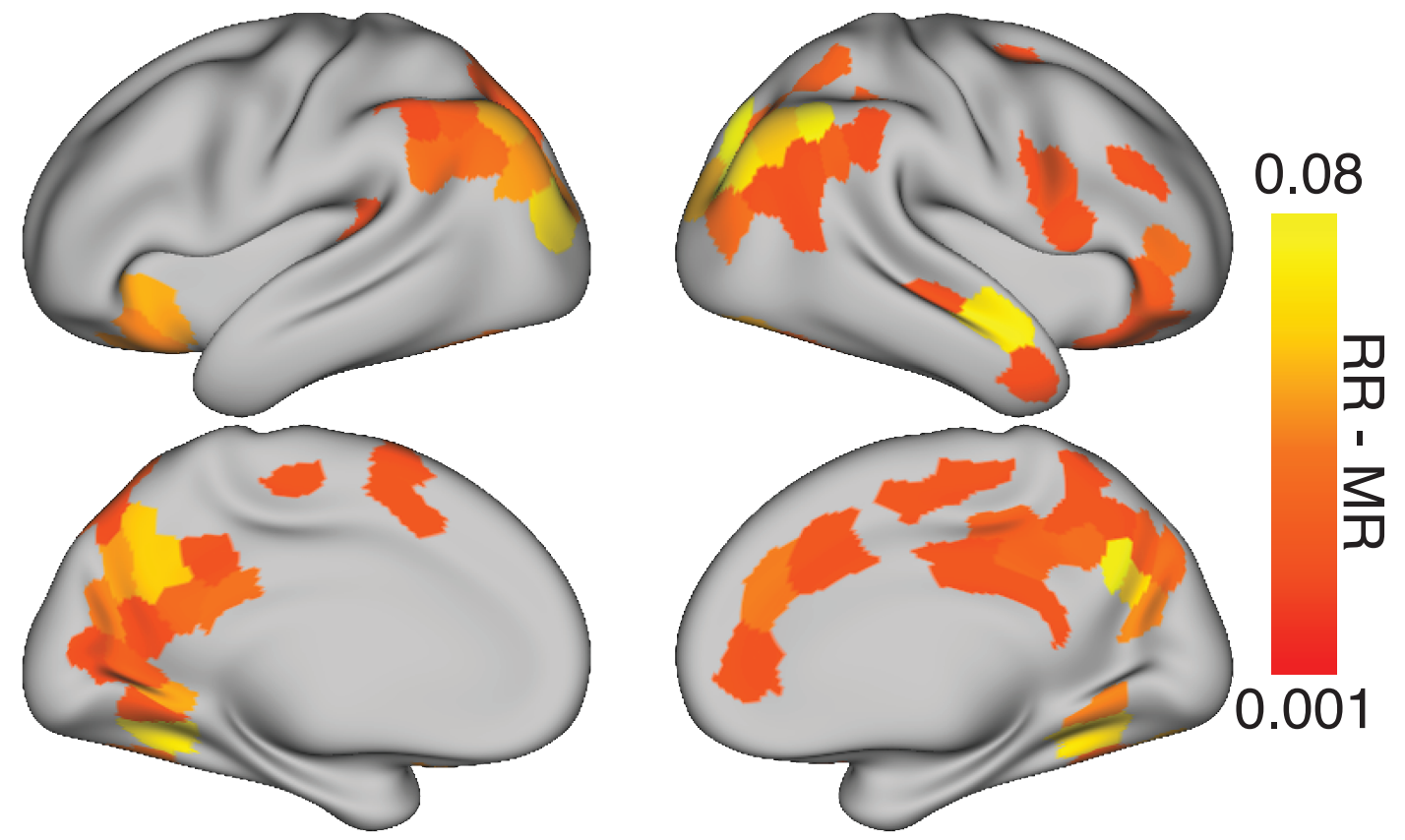

Supplementary Figure 2. Average memory transformation values (recall-to-recall minus movie-to-recall) from the searchlight analysis reported in Chen et al. 2017, shown in parcels where either recall-to-recall or movie-to-recall (between subjects) was reliable. For each reliable parcel (shown in Figure 3A, colored and light gray parcels), the memory transformation values observed in the parcel's searchlight centers were averaged together.

\begin{tabular}{|l|l|l|}
\hline Region of interest & $\begin{array}{l}\text { Schaefer parcel } \\
\text { ID }\end{array}$ & Notes \\
\hline $\begin{array}{l}\text { Posterior medial cortex } \\
\text { (PMC) }\end{array}$ & $154-160 ; 363-367$ & $\begin{array}{l}\text { All parcels labeled "PCC" in } \\
\text { "DefaultA" network }\end{array}$ \\
\hline
\end{tabular}

Supplementary Table 3. List of Schaefer 400 parcels used to create the posterior medial cortex (PMC) region of interest. 


\section{Supplementary Methods}

The behavioral and fMRI results described in the main manuscript were based on Direct Judgments of temporal precision, in which human raters labeled recall utterances based on whether the described events elapsed in more than ten seconds (Summary) or less than ten seconds (Temporally Precise). These labels can also be assigned with Automatic methods, as described below.

Indirect/Automatic: In a complementary analysis to the Direct Judgments, the categories of Summary and Precise were determined "automatically", based on the movie segment labels assigned to each statement, as judged by a coder who worked with the data prior to the current analysis (see Chen et al., 2017 and Vodrahalli et al., 2018 for details). This method differs from the Direct Judgment approach in that the Direct Judgment coders specifically considered whether each statement referred to a ten second window or longer, while the Automatic coder began by first segmenting the movie into 1000 events, then had the goal of assigning movie segments to each recall statement, without considering the unit of ten seconds. In the Automatic method, if the total summed duration of the labeled movie segments exceeded ten seconds, the statement was labeled as "Summary", otherwise it was labeled as "Precise." Utterances for which no corresponding movie segments could be identified were labeled as "Other."

Scene-level values of recall behavior can also be computed for each subject, akin to the Summary bias scores reported using the Direct Judgment method in the main manuscript. To compute these values, we repeated the steps described for Direct Judgment as follows: for each of the 50 movie scenes, we identified the recall utterances that described the scene (after translating the utterance-level movie event labels from the 1000 micro-segment level to the 50scene level). Then, we counted the number of words (excluding stop words) contained in these utterances, and calculated the proportion of words that came from utterances that had been automatically labeled as "Summary."

Temporal Compression Factor: The Indirect/Automatic approach also enabled us to calculate a "temporal compression factor" (TCF) for each recall utterance. For each utterance, we computed the number of spoken words (excluding stop words) and then divided this value by the total duration of the described movie events, which is the same duration described in the Automatic method above. Thus, the TCF value is a ratio, where the numerator quantifies the amount of information the participant devoted to describing the events, and the denominator quantifies how long it look these events to elapse in the movie. This value is subtracted from 1, so that higher TCF scores indicate increased temporal compression, or that fewer words were spent relative to the amount of time passed.

To compute scene-level TCF values for each subject, we identified the utterances that described each scene (scaling from the 1000 micro-segment level up to the 50-scene level), and then calculated the scene-average TCF value by averaging across the utterance-level TCF values.

Thus, the three different measures (Direct Judgment, Automatic, and Temporal Compression Factor) can each provide scene-level values of recall behavior for each subject (e.g., Summary bias scores for each scene, as described in the main manuscript). The three measures were positively correlated in each subject, as should be expected given that are variations of the same judgment (i.e., degree of temporal compression at recall relative to 
encoding). The Direct Judgment ratings of Summary bias are moderately correlated with both the Automatic ratings $(\mathrm{r}=0.55, S D=0.18, \min =0.19, \max =0.84)$ and the TCF scores $(\mathrm{r}=0.41, S D=$ $0.15, \min =0.11, \max =0.64)$, while the TCF and Automatic ratings are correlated at $\mathrm{r}=0.68$ $(S D=0.09, \min =0.51, \max =0.85)$. 